\title{
ASSESSMENT OF PHYSICIANS' HEALTH CARE NEEDS: IS THERE A LINK WITH QUALITY OF CARE IN SAUDI ARABIA?
}

\author{
Mohamed Mahrous ${ }^{1}$
}

${ }^{1}$ Associate Professor, Consultant of Quality in Health Care, Chairman of Medical Education Department, College of Dentistry, Taibah University Madinah, Saudi Arabia

Correspondence: mm67sa@yahoo.com Cell\#: +966555309009

\begin{abstract}
Background: In daily practice, physicians encounter many health risks. Multiple studies have found that physicians are finding it difficult to access health care, and often resort to self-diagnosis and treatment.

Methods: Cross-section analytical observational study. A non-random convenience sampling technique using a preexisting self-administered validated questionnaire.

Results: Most of the factors represented a low-to-moderate or neutral level of difficulty, although $42 \%$ of respondents said that finding the time to access care was a high priority. Career threatening illnesses were not a significant problem (72 of 456 respondents, or $15.8 \%$ ), but $54.4 \%$ of respondents reported that they had a colleague with a career-threatening illness.

Conclusion: The results indicate the need for policy makers and employer to make a greater effort to meet physicians' health care needs. One of the main weaknesses of this study was the sample, as it cannot be considered to be representative of physicians working in Saudi Arabia. The majority of respondents were general and family physicians, so the results may not apply to other specialties that were not represented equally in our sample.
\end{abstract}

Keywords: Doctors, physicians, healthcare, quality

\section{Introduction}

The well-being of health care providers is an important public health factor, as it is considered to be one of the predictors of quality health care. As they get older, one in two physicians will experience a major health problem (1). In general, physicians experience a high number of health risks $(2,3)$. Health care providers in general, and physicians in particular, are exposed to daily challenges, including irregular and long work hours (4), stress (including burnout syndromes), and chemical dependency, which, particularly in Western communities, may lead to suicide $(2,5)$.

Although financial constraints are not normally a real problem in many physician communities, a study showed that $53 \%$ of surveyed physicians reported that they had found it difficult to access health care, and $63 \%$ of respondents stated that they often resort to selfdiagnosis and treatment. Over $80 \%$ of the physicians surveyed either had, or knew, a colleague who had a career-threatening illness (4). Health care providers do not give their own health a great deal of consideration, either due to time constraints, or to issues relating to confidentiality (4); physicians experiencing psychological problems are at a higher risk of dying from suicide than the general population (2). It is clear that, when physicians are patients, it is extremely challenging for them to access the care that they need (5).

Another study pointed out that, although $95 \%$ of physicians agreed that working while sick had a serious impact on patients, $83 \%$ of them mentioned that they work while sick for in order to provide patients with continuity of care (6). Study participants offered health services whilst experiencing serious symptoms, such as diarrhoea, fever, and acute onset of significant respiratory symptoms (6). Once a physician experiences any medical condition, the consequential effect on efficiency can be noticed on both the individual and the health care system (7). Proper patient counselling is an outcome of physicians who practise healthy behaviours (8).

In fact, these challenges tend to affect physicians' ability to provide quality care, and lead to unsatisfactory patient outcomes.

Another factor that may contribute to the lack of health care services available to physicians is the fact that physicians tend to fear illness, as it may lead to weakness, an inability to perform their roles, and a loss of confidentiality and privacy (4).

Many physicians do not have a primary health care provider (9). However, the nature of their job gives many physicians easy access to medical care $(10,11)$. Gross et al. (9) found that $77 \%$ of physicians did not have a 
regular source of medical care, and another study on Australian physicians found that only $42 \%$ had a primary care provider (10).

A study of Canadian physicians demonstrated that physicians neglect their health: in the two years prior to the study, only $55 \%$ of physicians had attended a regular check-up (11). Physicians have a tendency to selfprescribe. One major obstacle to obtaining care may be the fact that physicians are unaware of sources of physical and mental health care, particularly if they have a substance abuse problem (11). A lack of training in offering medical services to physicians may also be a barrier to physicians offering care to their colleagues (5, 12).

The previous view was not addressed among physician in Saudi Arabia. This study aims to identify barriers faced by physicians when accessing health care services, and to explore the relationships between these barriers and demographic factors, such as the gender and working hours of the physicians. Furthermore, this study will identify physicians' health care needs and common medical conditions that may have affected their medical careers. All of these barriers will be discussed on the basis of how the quality of health care can be affected, given that those physicians practise health care.

\section{Methodology}

This study takes the form of a cross-sectional analytical observational study. The sample was obtained using a non-random convenience sampling technique.

With regard to the study tool, a literature review revealed that a validated survey addressing the health care needs of physicians or other similar professional groups already existed $(4,13)$. The survey was distributed to physicians attending continuing medical education conferences in various cities in Saudi Arabia over a sixmonth period, from July 2016 to December 2016. The survey was collected without personal identifiers. The original questionnaire consisted of 11 questions. The responses to these questions, which were given using a ten-point scale, will be summarized using means and standard deviations.

The first four questions were categorized into three groups based on the scores given by the respondents (14, low; 5-6, neutral; 7-10, high) and were analysed as a categorical variable. The remaining questions were analysed as continuous variables. $\mathrm{P}<0.05$ was considered statistically significant.

For the most common diseases, answers will be divided into six main groups: (i) psychiatric illnesses, including depression, anxiety, and schizophrenia; (ii) abuse of substances, including alcohol, cocaine, and other drugs and illegal substances; (iii) cognitive problems, including Alzheimer's disease, memory disturbance, and dementia; (iv) cancers of any kind; (v) neurological disorders, including paralysis, stroke, and Parkinson's disease; and (vi) heart diseases, including coronary artery disease, angina, and myocardial infarction. Responses between 1 and 4 on the Likert scale were considered to be negative, responses of 5 and 6 were considered to be neutral, and responses between 7 and 10 were considered to be positive.

Working hours were divided into three main groups: $<40$, 40-59, and >60 h per week, and the ANOVA test was used to explore the relationship between working hours and other variables. The T-test was used to explore the relationship between gender and other scale variables. For each of the barriers to getting appropriate health care, Pearson's correlation was performed in order to assess the correlation between experiencing difficulty in accessing health care and the likelihood of selfdiagnosis. The results will be presented in the form of coefficients, $95 \%$ confidence intervals, and P-values.

Inclusion criteria: Registered physicians working in the Kingdom of Saudi Arabia and willing to participate voluntarily in the survey

Ethical considerations: This study was revised and approved by the Research Ethics committee of the College of Dentistry, Taibah University (TUCD REC). A request for the waiver of the requirement to obtain consent forms was approved, since this study relies on a self-administered anonymous questionnaire.

\section{Results}

Over a period of six months, 750 questionnaires were distributed to physicians attending medical conferences; the distribution took place on an almost weekly basis. Four hundred and fifty-six (456) completed questionnaires were returned to the investigator, representing a response rate of $60.8 \%$. Almost twothirds $(222 / 456$, or $63.2 \%)$ of the study participants were male, whereas females accounted $168 / 456$, or $36.8 \%$ of the study participants. The most common specialty was general practice/family medicine (216/456, or $47.4 \%$ of participants); this was followed by medical subspecialties (128/456, or $28.1 \%$ of participants), surgical specialties (48/456, or $10.5 \%$ of participants), and other specialties, including laboratories, radiology, and physical medicine (64/456, or $14 \%$ of participants). With regard to working hours, approximately half of the study participants reported that they worked for between 40 and 60 hours per week, 136 of the 456 participants (29.8\%) reported that they worked for fewer than 40 hours per week, and 64 participants (14\%) reported that they worked for more than 60 hours per week. Thirty five percent (160/456) of respondents felt that it was difficult to find the time to access care, but $72 \%$ (328/456) stated that they resorted to self-diagnosis and treatment because of the barriers to accessing care. With regard to those barriers, most of the factors represented a low-tomoderate or neutral level of difficulty, although 192 participants $(42 \%)$ claimed that finding the time to access care was the most significant barrier. Careerthreatening illnesses, which were reported by 72/456 participants $(15.8 \%)$, did not constitute a significant problem, although $54.4 \%$ of participants reported having a colleague with a career-threatening illness. Fifty-six percent (256/456) of our study participants reported that, 
on at least one occasion, they had needed to refer an ill colleague.

As shown in Table 1, the study participants were asked to use a five-point Likert scale to prioritize the illnesses that they perceived to affect health care providers (physicians in general). The results indicate that psychiatric illnesses and substance abuse may be the highest priorities, although their means were $3 \pm 1.4$ and $2.8 \pm 1.5$, respectively. Neurological and cognitive disorders followed closely behind, whereas cancer and heart disease were the illnesses that were perceived to be least likely to affect health care providers.

Table 1: IIInesses that are perceived to affect physicians' ability to practice

\begin{tabular}{|l|c|c|}
\hline \multicolumn{1}{|c|}{ Illness } & Mean & SD \\
\hline Psychiatric Illness & 3 & 1.48 \\
\hline Substance abuse & 2.86 & 1.5 \\
\hline Cancer & 2.66 & 1.2 \\
\hline Heart diseases & 2.43 & 1.17 \\
\hline Neurological/cognitive disorders & 2.73 & 1.34 \\
\hline
\end{tabular}

\section{Access to healthcare services}

Table 2 shows the responses to the main survey questions; these responses have been grouped according to the gender of the respondents and the number of hours worked per week. There was a statistically significant difference between male and females regarding difficulty in accessing health care: female participants experienced more difficulties than males $(P<0.001)$. On the other hand, the results indicate that male practitioner are more likely to resort to selfdiagnosis or self-treatment than their female counterparts $(P=0.001)$.

With regard to the main barriers, statistically significant differences between male and female participants were observed in relation to their ability to find the time to access care, the cost of care, the cost of the lost work time, and the fact that they were not encouraged to access care by their employer $(P<0.001)$. No statistically significant difference was observed between male and female participants regarding confidentiality and the issue of seeing someone they know. When the results were sorted according to working hours, it was found that those who work more than 60 hours per week had lower mean of perceiving difficulties in accessing care and were most likely to resort to self-diagnosis/self-treatment $(\mathrm{P}<0.001 \mathrm{for}$ both items). Difficulties in finding the time to access care were highest among those who work more than 60 hours per week $(P<0.01)$. Not being encouraged by one's employer was not associated with working hours $(p=0.36(N S))$.

\section{Reported barriers}

Table 4 demonstrates the correlation between experiencing difficulty in accessing health care, the likelihood of self-diagnosis/self-treatment, and the perceived barriers. There is a statistically significant correlation between experiencing difficulty in accessing to health care and experiencing difficulty in finding the time to access health care $(r=-0.129, P=0.006)$ and also between experiencing difficulty in accessing health care and not being encouraged by one's employer $(r=-0.228$ $\mathrm{P}=<0.001$ ). A similar tendency was found with other barriers, but this link was not found to be significant. $A$ positive statistical correlation was noticed between all barriers and the likelihood of self-diagnosis or selftreatment $(P<0.001)$. Thus, physicians who resorted to self-diagnosis were the most likely to be affected by all of the barriers $(P<0.001$ for all barriers).

Table 2: Association between experiencing difficulty in accessing health care, self-diagnosis, and the perceived barriers to accessing care

\begin{tabular}{|c|c|c|c|c|c|c|}
\hline $\begin{array}{l}\text { Main barriers to } \\
\text { physicians getting } \\
\text { appropriate health } \\
\text { care? }\end{array}$ & & $\begin{array}{l}\text { Difficulty in } \\
\text { accessing } \\
\text { health care }\end{array}$ & & & \begin{tabular}{|l} 
Likelihood of \\
self- \\
diagnosis/self- \\
treatment
\end{tabular} & \\
\hline & Coef. (r) & $95 \%$ (CI) & $\begin{array}{c}\text { P- } \\
\text { Value }\end{array}$ & $\begin{array}{c}\text { Coef. } \\
\text { (r) }\end{array}$ & $95 \%$ (CI) & P-Value \\
\hline Finding time & $(-0.129)$ & $(-214)-(-0.36)$ & 0.006 & 0.34 & \begin{tabular}{|l|}
$0.27-0.41$ \\
\end{tabular} & $<0.01$ \\
\hline Confidentiality & 0.076 & \begin{tabular}{|l|}
$(-0.009)-0.16$ \\
\end{tabular} & 0.103 & 0.135 & \begin{tabular}{|l|}
$0.051-0.215$ \\
\end{tabular} & 0.041 \\
\hline $\begin{array}{l}\text { Seeing someone I } \\
\text { know }\end{array}$ & 0.073 & \begin{tabular}{|l|}
$(-0.2)-0.169$ \\
\end{tabular} & 0.122 & 0.162 & \begin{tabular}{|l|}
$0.082-0.237$ \\
\end{tabular} & 0.001 \\
\hline Cost of care & $(-0.119)$ & $\begin{array}{l}(-0.217)-(- \\
0.31)\end{array}$ & 0.11 & 0.151 & $0.58-0.246$ & 0.001 \\
\hline $\begin{array}{l}\text { Cost of lost work } \\
\text { time }\end{array}$ & 0.011 & \begin{tabular}{|l|}
$(-0.08)-0.098$ \\
\end{tabular} & 0.812 & 0.368 & \begin{tabular}{|l|}
$0.295-0.44$ \\
\end{tabular} & $<0.001$ \\
\hline $\begin{array}{l}\text { Not encouraged by } \\
\text { employer }\end{array}$ & $(-0.228)$ & $\begin{array}{l}(-0.317)-(- \\
0.138)\end{array}$ & $<0.001$ & 0.271 & \begin{tabular}{|c|}
$0.33-0.473$ \\
\end{tabular} & $<0.001$ \\
\hline
\end{tabular}

Figures 1 gives participants' responses to questions about barriers to care on a scale of $1-10$, where 1 is a minor barrier and 10 is a major one. Of all the perceived barriers to physicians obtaining health care, finding time had the highest median, whereas the cost of care and concerns about confidentiality were the barriers with the lowest medians.

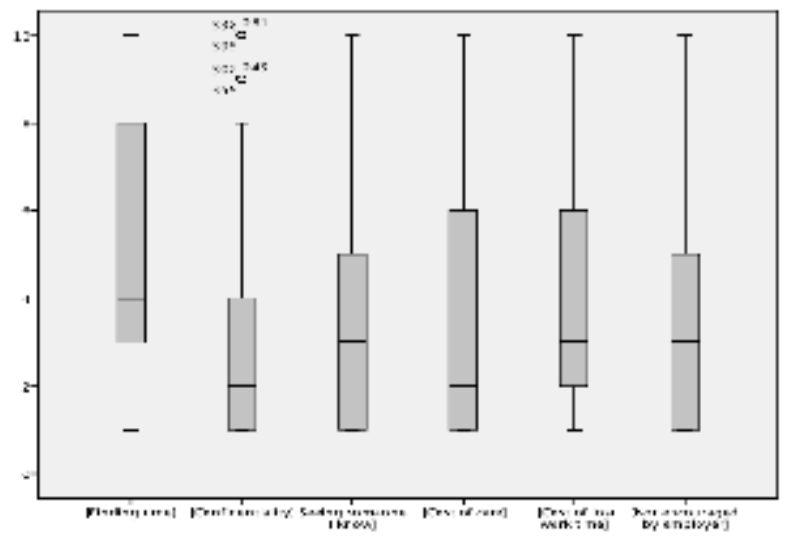

Figure 1: The main barriers that physicians experience when trying to access health care 
Figure 2 presents participants' responses to questions about the usefulness of services, where 1 is not useful at all and 10 is very useful. Rapid referral, access to comprehensive care, and rapid walk-in access to preventive care had the highest medians $(6.67,7$, and 7 , respectively), and were the most commonly cited factors in relation to the usefulness of services.

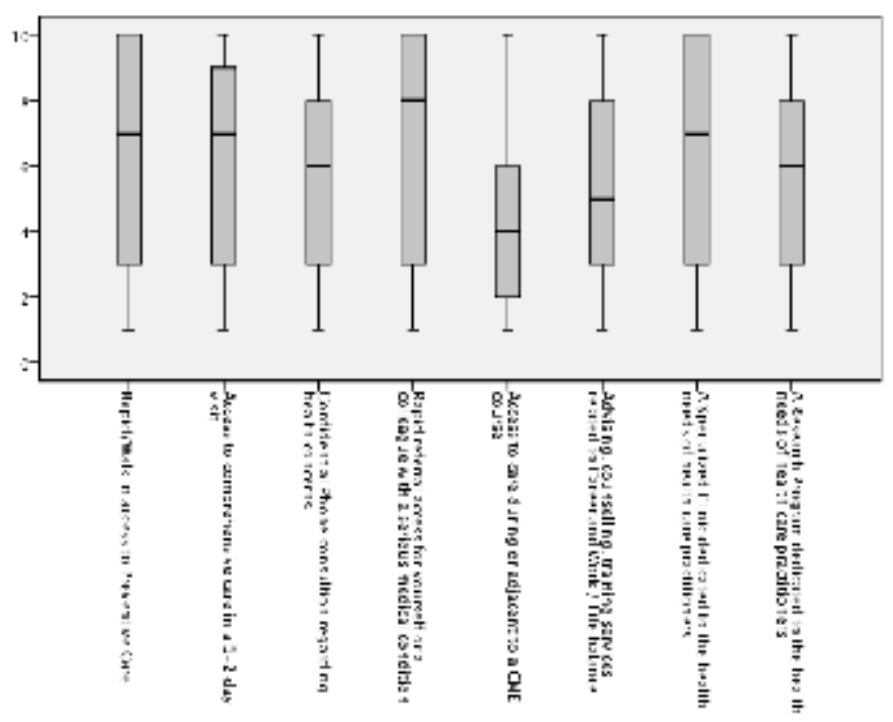

Figure 2:The usefulness of service to physicians Experience of illness and suggested services

As shown in Table 1, approximately $16 \%$ of respondents had experienced a career-threatening illness on at least one occasion, and $54.4 \%$ of respondents personally knew a colleague who had a career-threatening illness. Around $53 \%$ of participants stated that they had been in the position of wondering whether or not a colleague's illness made them unsafe to practise medicine. Furthermore, $56.1 \%$ of participants reported that they had needed to refer an ill colleague for assessment.

\section{Discussion}

Physicians are the most important factor in proper health care delivery. As can be seen in Table 1, the present study clearly shows that physicians still find it difficult to find the time to seek out health care for themselves, and also find it difficult to get access to appropriate health care services. As a result of barriers to health care, such as the ability to find the time, the majority of physicians tend to resort to self-diagnosis and treatment. These results were expected, as physicians are fully engaged with their patient and want to give them the best quality care they can. Consequently, they find it difficult to find the time to access health care for themselves. This was in accordance with the findings of the other study, in which $53 \%$ of the physicians surveyed reported having difficulty in finding time for health care and accessing health care, and $63 \%$ often resorted to self-diagnosis and treatment (4). Another study, which was conducted in the USA, also indicated that physicians still find it difficult to access health care services, due to barriers such as not being able to find the time (13). Moreover
Table 1 clearly indicates that career-threatening illnesses are not a significant problem (16\%). Nevertheless, $54.4 \%$ of participants reported having a colleague with a career-threatening illness, and this figure matches the results of the other study (13). Fiftysix percent of our study participants have needed to refer an ill colleague, and this result supported our belief that, as a result of the barriers to accessing care, the majority of physicians who become patients are referred by one of their colleagues.

With regard to the illnesses that respondents perceived as having an effect on physicians' ability to practise, Table 2 shows that psychiatric illness and substance abuse were perceived as having the greatest effect. Neurological/cognitive disorders were in second place, whereas cancer and heart diseases were perceived to have the least effect on physicians' ability to practise. These finding correspond to those of another study (4, 12).

When the main survey questions were grouped by gender and the number of hours worked per week, the results, which are shown in Table 3, indicate that female physicians reported having more difficulties in accessing health care than their male counterparts; this gender difference was also apparent in another study (13). In this regard, it is interesting to note that the emotional behaviours of female physicians concerning their patients may have a greater effect on their ability to access health care than those of their male colleagues.

Furthermore, the findings reveal that male physicians have a greater tendency to self-diagnose and self-treat than females. When the results for the main barriers are broken down by gender, some statistically significant differences between male and female participants were observed. These barriers include finding time, the cost of care, the cost of lost work time, and a lack of encouragement to seek health care on the part of their employer. All of these factors were perceived to be more significant barriers by males than females. This could be due to the fact that males, by nature, are used to working, and tend to continue to do so, thus creating barriers that prevent them from seeking health care.

When working hours were taken into consideration, it was found that those who worked more than 60 hours per week had a lower mean of perceiving difficulties in accessing care and also had the highest likelihood of resorting to self-diagnosis/self-treatment. Difficulties in finding the time for health care were most common among those who worked more than 60 hours per week. These results support the findings of another study, which also found that those who work more than 60 hours per week were most likely to resort to selfdiagnosis/self-treatment and were most likely to have difficulties in finding the time for their own health care needs (13). These results were not unexpected, as it is clear that those who work longer hours will have less time available for seeking health care, and will, therefore, resort to self-diagnosis or self-treatment. 
The associations between perceived barriers and a physician's willingness to access health care, and the likelihood of resorting to self-diagnosis or self-treatment, are shown in Table 4 . In the present study, physicians who experienced difficulties in accessing healthcare were more likely to face barriers, such as problems finding enough time for their own care, and a lack of encouragement by their employer. The findings indicate that physicians who resort to self-diagnosis also face these barriers; this association was statistically significant for all the barriers mentioned above. These all suggest that there would be a greater effect on the quality of services provided by those physicians, as those barriers would, in general, be likely to affect a physician's ability to provide quality care and achieve high levels of patient satisfaction. These results also similar to other study results (13). There is an urgent need for employers and policy makers to make significant changes and to increase physicians' willingness to consider their own health needs.

The results show that finding time to access care was the highest ranked barrier to getting care Ffigure1), while rapid referral access for serious medical conditions, access to comprehensive care within 1-2 days, and rapid/walk-in access to preventive care were most commonly cited as very useful (Figure 2). Those findings match those of the other study (4). These findings stress the need for physicians to have the right to access care, and the need for a system of rapid referral and the provision of comprehensive care within 1-2 days, in addition to the need for rapid walk-in access to preventive care. In Saudi Arabia, physicians and other health care providers access care in a similar manner to the general population. It is true here when it compared with physicians face finding time as a barrier for them to acquire health needs so the availability of the mentioned condition could do the balance. In fact, this balance can be translated to measured outcomes, and named as quality care given to patients.

In this study, a significant percentage of respondents stated either they had, or knew, a colleague with a career-threatening illness. Around 16\% of respondents reported experiencing a career threatening illness at least once, and $54.4 \%$ personally knew a colleague who had such an illness. Approximately $53 \%$ of respondents reported that they had been in a situation where they have wondered whether an ill colleague was safe to practise medicine. In addition, $56.1 \%$ of respondents reported that they had needed to refer an ill colleague for assessment. These findings give rise to great concerns about quality of care: once physicians experience any medical condition, the consequential effect on efficiency can be noticed on both the individual and the health care system (7). Proper patient counselling is an outcome of physicians who exercise healthy behaviours (8). These findings match those of other studies $(4,14)$.

The feedback obtained from the free text comments appears to match the results for the study as a whole, as some respondents confirmed that physicians are the worst type of patients, as they face barriers to accessing care and also work while they are sick. Respondents also suggested having private medical insurance that is partially financed by the workplace. Others believed that annual medical check-ups should be mandatory. They also highlighted the need for raising awareness of wellbeing and work-life balance among health care workers, and the need for policies on mental health, cancer, and other health risks. Quick access to medical care for physicians is a priority, as this will encourage them to take care of themselves. One important comment made in the free text comments section stressed that not all physicians know that caring for their health is a part of their professionalism. In my opinion, all of the comments above make sense, and lead us to believe that physicians and other health care providers need special consideration in order to take care of themselves and to ensure that the care that they provide is of high quality and leads to high rates of patient/family satisfaction.

\section{Conclusion}

Physicians report that they face significant barriers when trying to access health care services for themselves. In fact, female physicians experience greater difficulty in accessing care that their male counterparts. The barriers identified in this study are subject to modification and rearrangement. The findings indicate that policy makers and employers need to make a greater effort to improve the health of physicians.

\section{References}

1. Harms BA., Heise CP., Gould JC., Starling JR. A 25-year single institution analysis of health, practice, and fate of general surgeons. Ann Surg. 2005;242(4):520-6. discussion 526-529.

2. Schernhammer ES, Colditz GA. Suicide rates among physicians: a quantitative and gender assessment (meta-analysis). American Journal of Psychiatry. 2004;161(12):2295-302.

3. Frank E, Brogan DJ, Mokdad AH, Simoes EJ, Kahn HS, Greenberg RS. Health-related behaviors of women physicians vs other women in the United States. Archives of Internal Medicine. 1998;158(4):342-8.

4. Steffen M, Hagen P, Benkhadra K, Molella RG, Newcomb RD, Murad MH. A survey of physicians' perceptions of their health care needs. Occupational Medicine. 2015;65(1):4953.

5. Schneck SA. Doctoring doctors and their families. Jama. 1998;280(23):2039-42.

6. Szymczak JE, Smathers S, Hoegg C, Klieger S, Coffin SE, Sammons JS. Reasons why physicians and advanced practice clinicians work while sick: a mixed-methods analysis. JAMA pediatrics. 2015;169(9):815-21.

7. Davis WT., Sathiyakumar V., Jahangir AA., Obremskey WT., Sethi MK. Occupational injury among orthopaedic surgeons. J Bone Joint Surg 
Am2013;95:e107.

8. Vickers KS., Kircher KJ., Smith MD., Petersen LR., Rasmussen NH. Health behavior counseling in primary care: provider-reported rate and confidence. Fam Med. 2007; 39(10):730-5.

9. Gross CP, Mead LA, Ford DE, Klag MJ. Physician, heal thyself?: regular source of care and use of preventive health services among physicians. Archives of Internal Medicine. 2000;160(21):3209-14.

10. Pullen D, Lonie CE, Lyle DM, Cam DE, Doughty MV. Medical care of doctors. The Medical Journal of Australia. 1995;162(9):481, 4-, 4.

11. Frank E, Segura C. Health practices of Canadian physicians. Canadian Family Physician. 2009;55(8):810-1. e7.

12. Tyssen R. Health problems and the use of health services among physicians: a review article with particular emphasis on Norwegian studies. Industrial health. 2007;45(5):599-610.

13. Benkhadra K, Adusumalli J, Rajjo T, Hagen PT, Wang Z, Murad MH. A survey of health care needs of physicians. BMC Health Services Research. 2016;16(1):472.

14. DesRoches CM., Rao SR., Fromson JA. Physicians' perceptions, preparedness for reporting, and experiences related to impaired and incompetent colleagues. J Am Med Assoc. 2010; 304:187-193. 